\title{
RESTITUZIONE DI PRIORITÀ.
}

\section{Nota di Gaetano Scorza (Parma).}

\author{
Adunanza del 28 dicembre 1913 .
}

La formula che nella Nota: Sui determinanti emisimmetrici d'ordine pari e sui relativi pfaffiani [questi Rendiconti, tomo XXXVI ( $2^{\circ}$ semestre 1913), pp. I71-176] ho dedotto come applicazione dei teoremi stabiliti nelle mie: Osservazioni varie sulla teoria delle sostitu ioni e sulle parti-ioni dei numeri interi in numeri interi [Ibid., id., pp. 163-170] era stata già incontrata dal prof. U. SCARPIs nella sua Nota: Una proprietà dei determinanti dedotta dal concetto di sostituzione [Giornale di Matematiche di Battaglini, vol. XXXVII (1899), pp. 73-79].

Parma, 18 dicembre 19I3.

GaETANO SCORZA.

\section{ERRATA-CORRIGE}

Avvertenza. - Le linee si contano dall'alto della pagina escludeudovi la intestatura.

I. -Errorisfuggitiagli Autorinella revisionedelle bozzedi stampa:

Toxo Pagina Limea In Luogo di: LeGgere:

$\begin{array}{ccccc}\text { XXXVI } & 381 & 3,7,18 & \left(\varphi\left(y^{\prime}\right)\right)^{2} & \varphi\left(y^{\prime}\right) \\ \text { XXXVII } & 281 & 18 & \text { HamilthoN } & \text { Hamilton } \\ » & 332 & 4 & \text { Cauchy and Binet } & \text { CauchY and BIN }\end{array}$

$\begin{array}{cc}\text { Tоuо } & \text { PagiNa } \\ \text { XXXVII } & 2 \\ " & 28 \\ " & 298 \\ " & 302\end{array}$

II. Errori tipografici:

LimEA

Io

32

I7

3 I
IX LUOGO DE :

Zahl

( ${ }^{\circ}$ semestre 1913)

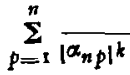

Sätze III
LEGGRE:

Zahl ( $2^{\circ}$ semestre 1913)

$\sum_{p=1}^{n} \frac{r}{\left|a_{n p}\right|^{k}}$

Satze III 